\title{
Factors Influencing Treatment Recommendations in Node-Negative Breast Cancer
}

\author{
By Elisabeth Edstrom Elder, MBBS, PhD, FRACS, Sally Baron Hay, MBBS, FRACP, and Katrina Moore, \\ MBBS, MS, FRACS
}

Royal North Shore Hospital, Sydney, Australia

\begin{abstract}
Purpose: To assess factors influencing recommendations for adjuvant chemotherapy (CT) in relation to perceived benefits in women with stage 1 breast cancer and to determine the degree to which recommendations were followed.

Methods: Recommendations from multidisciplinary team meetings at Royal North Shore Hospital (Sydney, Australia) during 2007 and 2008 for postoperative patients with invasive, primary breast cancer were reviewed. Treatment data were collected from patients' medical records. Estimated benefits of adjuvant CT on 10-year survival for node-negative patients were calculated using Adjuvant! Online. Univariate and multivariate analyses were performed using SPSS.
\end{abstract}

Results: Of 345 patients (mean age, 59 years), 51 were unsuitable for CT as a result of comorbidity and/or age $>80$ years. All 93 patients with nodal macrometastases who were suitable

\section{Introduction}

Early detection from mammographic screening and greater societal awareness of breast cancer risks have resulted in an increasing number of women being diagnosed with stage I breast cancer. At the same time, the indications for adjuvant chemotherapy have broadened to include patients with node-negative disease in the presence of other adverse features such as tumor size $>1 \mathrm{~cm}$, high grade, lack of estrogen receptor (ER) expression, and human epidermal growth factor receptor 2 (HER2) overexpression. ${ }^{1-3}$

Although adjuvant systemic therapy has greatly improved survival of patients with early-stage breast cancer, ${ }^{4}$ the absolute benefit of chemotherapy in this group is generally modest. The gain needs to be balanced against the risk of nontrivial adverse effects, including infection, heart failure, and leukemia, among others. ${ }^{2}$ The decision-making process of recommending chemotherapy is complex, involving assessment of the risk of recurrence versus the risk of treatment-related adverse effects. It is important to take the patient's perspective into consideration during this process.

Adjuvant! Online is a user-friendly, Web-based program that provides an estimate of the benefit of adjuvant systemic therapy on survival and risk of relapse, on the basis of individual patient information and tumor characteristics. The estimates are derived from results of a large number of trials involving thousands of patients in various populations. ${ }^{5}$ Adjuvant! Online is used by many clinicians as a valuable tool in patient counseling and as an aid for treatment recommendations in multidisciplinary meetings. for CT and 20 (80\%) of 25 with micrometastases were recommended for CT, compared with 92 (53\%) of 175 node-negative patients. Tumor size $>2 \mathrm{~cm}$, grade 3 , estrogen receptor negativity, and age less than 45 years were independent factors influencing CT recommendation. The mean estimated benefit of CT in node-negative patients who received this recommendation was $5.7 \%$ versus $1.3 \%$ in patients not recommended for CT. Twenty-one (23\%) node-negative patients declined CT after discussion with a medical oncologist. A higher proportion of nodenegative patients were recommended for CT in 2008 versus 2007 (60\% v 44\%, $P=.04)$.

Conclusions: Grade, tumor size, estrogen receptor status, and younger age are the most significant factors influencing CT recommendation in node-negative patients. The minimum level of benefit to recommend CT is approximately $2 \%$. A significant proportion of patients do not proceed with CT after individualized review.

The pattern of chemotherapy delivery varies greatly between institutions. A survey of the frequency of chemotherapy use for hormone receptor-positive, lymph node-negative breast cancer from 1997 to 2004 at eight National Comprehensive Cancer Network institutions in the United States showed that the rate of chemotherapy use varied between $46 \%$ and $65 \%$, with a change over time varying from a $79 \%$ relative increase to a $22 \%$ relative decrease. ${ }^{6}$

Recommendations for disease management in patients with cancer are made by our local multidisciplinary team (MDT) of breast surgeons, medical and radiation oncologists, radiologists, pathologists, trainees in these specialties, breast care nurses, and allied health personnel and covers patients treated in both the public and private sector. The current study aimed to assess factors that influence recommendations for adjuvant chemotherapy in node-negative patients in relation to perceived benefits, as well as to assess changes over time as part of an audit process. We also aimed to determine the degree to which recommendations of the MDT were followed and sought to assess the reasons if they were not.

\section{Methods}

A summary sheet including demographic data, clinical information, pathology, calculation of benefit per Adjuvant! Online, and recommendations for adjuvant therapy was created and collected prospectively for each patient discussed at the weekly MDT meeting at Royal North Shore Hospital. We then reviewed these summary sheets for all postoperative patients with breast cancer discussed between January 1, 2007, and December 
31, 2008. Patients with recurrent disease, patients with ductal carcinoma in situ without any invasive component, and those who had received neoadjuvant chemotherapy or had unknown nodal status were excluded.

The data on the MDT sheets were verified by information from pathology providers and from patients' files. Histopathology was reported according to the American Joint Committee on Cancer guidelines. ${ }^{7}$ Information on acceptance of chemotherapy was collected from patients' files and records from medical oncology departments. For patients with node-negative disease, the benefit of adjuvant chemotherapy in 10-year survival and risk of relapse was calculated using Adjuvant! Online (version 8.0), on the basis of verified clinical and pathological information, assuming use of a second-generation chemotherapy agent. (In Australia, government funding is presently not available for third-generation chemotherapy agents in nodenegative, HER2-negative, early-stage breast cancer.) Fitness for chemotherapy was assessed clinically, taking into account the patient's perceived biologic rather than chronological age. For the purpose of calculating an estimated benefit of chemotherapy, the patient's level of comorbidity was classified according to the Adjuvant! Online program's help files. 5

Statistical analyses were performed using SPSS (version 15.0). The level of statistical significance was set at $P<.05$. Correlation between categorical variables was analyzed by $\chi^{2}$, and between continuous variables with the Student $t$ test. Variables that significantly influenced outcome in the univariate analyses were entered into a multivariate logistic regression analysis using both backward and forward stepwise models.

\section{Results}

\section{Study Population}

A total of 448 postoperative patients with breast cancer were discussed at the Royal North Shore Hospital MDT meetings between January 1, 2007, and December 31, 2008. For 92\% of the patients, surgery was performed by two surgeons who both presented all their breast cancer cases in this forum. Patients with recurrent disease $(\mathrm{n}=26)$, ductal carcinoma in situ without any invasive component $(\mathrm{n}=62)$, and unknown nodal status $(\mathrm{n}=2)$, as well as male patients $(\mathrm{n}=1)$ and those who had received neoadjuvant chemotherapy $(\mathrm{n}=11)$ were excluded, leaving 345 patients. The two patients with unknown $N$ stage did not have axillary surgery because of advanced age and multiple comorbidities. Both of these patients were clinically node negative. The mean age of the 345 patients in the study group was 59 years, with 53 patients being 45 years or younger and 37 patients older than 80 years.

Standard surgical treatment was breast-conserving surgery or mastectomy (with or without immediate breast reconstruction) and sentinel node-based assessment of the presence of lymph node metastasis. There was no difference in treatment patterns between patients treated in the public or private sector (one third $v$ two thirds, respectively).

All patients who underwent breast-conserving surgery were recommended for whole-breast radiotherapy. Postmastectomy radiotherapy was recommended to women with a high risk of locoregional recurrence as determined by primary tumor characteristics and the number of involved lymph nodes. All patients with hormone receptor-positive disease were considered for hormonal adjuvant therapy with either tamoxifen or an aromatase inhibitor, on the basis of their menopausal status and the potential adverse effects of these drugs. Patients considered for chemotherapy were usually recommended an anthracyclinebased regimen, followed by a taxane in patients with nodepositive disease. Patients with HER2-positive disease were generally recommended trastuzumab in conjunction with chemotherapy. ${ }^{8}$ Participation in a clinical trial was encouraged when appropriate.

\section{Factors Influencing Recommendation of Adjuvant Chemotherapy}

The most significant factor influencing recommendation of adjuvant chemotherapy was nodal status. There were 108 patients (31\%) with nodal macrometastases (nodal tumor deposit(s) $>$ $2 \mathrm{~mm}$ ); 29 patients (9\%) with micrometastases (tumor deposit $0.2-2 \mathrm{~mm}$ ); and 208 patients $(60 \%)$ considered to have nodenegative disease, including 14 with isolated tumor cells $(<0.2$ $\mathrm{mm}$ ) in the lymph nodes. Of these, 51 patients $(15 \%)$, equally distributed between the three groups, were assessed as not being suitable for chemotherapy as a result of either significant comorbidities or frailty associated with old age (usually $>80$ years). One patient was not eligible for chemotherapy because there had been a $>3$ month interval between her initial lumpectomy and the axillary dissection showing nodal involvement (Table 1).

All of the 93 eligible patients with nodal macrometastases who were considered suitable were recommended for chemotherapy. Of the 25 patients with nodal micrometastases who were suitable, 20 (80\%) were recommended for chemotherapy. The five patients who were not recommended for chemotherapy were all discussed at MDT meetings during 2007. Their tumors had low risk features, that is, size less than $15 \mathrm{~mm}$, low or intermediate grade, estrogen-receptor positive, and HER2receptor negative. Patients with micrometastases and similar tumor characteristics discussed during 2008 were recommended for chemotherapy.

Ninety-two (53\%) of the 175 suitable patients who were considered node negative were recommended for chemotherapy, including 10 of the 13 patients with isolated tumor cells in the sentinel node (Nis + disease; all of these 10 patients had a primary tumor $>2 \mathrm{~cm}$ in diameter; Table 1). For patients with low estimated benefit, the MDT modified the recommendation to a detailed discussion with a medical oncologist regarding benefits and risks.

Factors influencing chemotherapy in the 175 node-negative patients were analyzed in more detail. By univariate analysis, chemotherapy was influenced by tumor grade $(P<.001)$, T stage $(P<.001)$, age less than 45 years $(P<.001)$, ER status $(P<.001)$, HER 2 status $(P<.002)$, and the presence of lymphovascular invasion $(P<.03$; Table 2$)$. Tumor grade, tumor size, ER status, and age less than 45 years remained 
Table 1. Nodal Status and Adjuvant Chemotherapy Recommendation

\begin{tabular}{|c|c|c|c|c|c|c|}
\hline \multirow[b]{2}{*}{ Nodal Status } & \multirow[b]{2}{*}{ No. } & \multirow[b]{2}{*}{$\%$} & \multirow[b]{2}{*}{$\begin{array}{l}\text { Not Suitable for } \\
\text { Chemotherapy }\end{array}$} & \multirow[b]{2}{*}{$\begin{array}{l}\text { Suitable for } \\
\text { Chemotherapy }\end{array}$} & \multicolumn{2}{|c|}{$\begin{array}{l}\text { Recommended } \\
\text { Chemotherapy }\end{array}$} \\
\hline & & & & & No. & $\%$ \\
\hline Micrometastases & 29 & 9 & 4 & 25 & 20 & 80 \\
\hline Macrometastases & 108 & 31 & 14 & 94 & 93 & 99 \\
\hline Total & 345 & 100 & 51 & 294 & 205 & 70 \\
\hline
\end{tabular}

* Including 14 patients with nodal isolated tumor cells: 10 of these patients were recommended for chemotherapy.

significant in multivariate analysis. Menopausal status did not significantly influence chemotherapy. Grade, ER status, and age less than 45 years were significant factors in the 83 nodenegative patients with a T1c tumor (1 to $2 \mathrm{~cm}$ ).

Table 2. Factors Influencing Recommendation for Adjuvant Chemotherapy in Univariate Analyses of 175 Patients Who Were Suitable for Chemotherapy

\begin{tabular}{|c|c|c|c|}
\hline Factor & $\begin{array}{l}\text { Chemotherapy } \\
\text { Recommended } \\
(\mathrm{n}=92)\end{array}$ & $\begin{array}{l}\text { Chemotherapy Not } \\
\text { Recommended } \\
\text { (n=83) }\end{array}$ & $P$ \\
\hline \multicolumn{4}{|l|}{ Grade } \\
\hline 1 & 3 & 42 & $<.001$ \\
\hline 2 & 34 & 36 & \\
\hline 3 & 55 & 5 & \\
\hline \multicolumn{4}{|l|}{ T stage } \\
\hline $1 \mathrm{a}(<0.5 \mathrm{~cm})$ & 2 & 15 & $<.001$ \\
\hline $1 \mathrm{~b}(0.5-1 \mathrm{~cm})$ & 2 & 21 & \\
\hline $1 \mathrm{c}(1-2 \mathrm{~cm})$ & 41 & 42 & \\
\hline $2(2-5 \mathrm{~cm})$ & 43 & 5 & \\
\hline $3(>5 \mathrm{~cm})$ & 4 & 0 & \\
\hline Age, years & & & $<.001$ \\
\hline$<45$ & 24 & 4 & \\
\hline$>45$ & 68 & 79 & \\
\hline \multicolumn{4}{|l|}{ ER status } \\
\hline Positive & 61 & 83 & $<.001$ \\
\hline Negative & 31 & 0 & \\
\hline \multicolumn{4}{|l|}{ HER2 status } \\
\hline Positive & 15 & 2 & .002 \\
\hline Negative & 77 & 81 & \\
\hline \multicolumn{4}{|l|}{ LVI status } \\
\hline Positive & 33 & 13 & .03 \\
\hline Negative & 57 & 67 & \\
\hline Nodal status & & & .06 \\
\hline Negative & 82 & 80 & \\
\hline Isolated tumor cells & 10 & 3 & \\
\hline Pre/perimenopausal & 39 & 25 & .09 \\
\hline Postmenopausal & 53 & 58 & \\
\hline Discussed in 2007 & 36 & 45 & .04 \\
\hline Discussed in 2008 & 56 & 38 & \\
\hline
\end{tabular}

Abbreviations: ER, estrogen receptor; HER2, human epidermal growth factor receptor 2; LVI, lymphovascular invasion.

\section{Benefit of Chemotherapy Per Adjuvant! Online and Outcome of the Recommendation}

In patients with node-negative disease who were recommended for chemotherapy, the average benefit indicated by Adjuvant! Online was a $5.7 \%$ (range, $1.5 \%$ to $17.3 \%$ ) reduction in 10 year mortality and an average $11.8 \%$ (range, $4.8 \%$ to $24 \%$ ) reduction in the 10-year risk of relapse. In contrast, the average benefit in mortality was $1.3 \%$ (range, $0.1 \%$ to $3.7 \%$ ) and in relapse, $6.1 \%$ (range, $2.4 \%$ to $11.5 \%$ ) in node-negative patients who were not recommended for chemotherapy (Fig 1).

Twenty-one (23\%) of 92 patients without nodal involvement who were recommended for chemotherapy did not proceed to chemotherapy. In 14 of these patients, this was because the medical oncologist did not recommend chemotherapy at the individual consultation subsequent to the MDT meeting. The average benefit in 10-year survival indicated by Adjuvant! Online in this group was 3.5\% (range, $1.6 \%$ to $7 \%$ ) and $8.5 \%$ (range, $6 \%$ to $16.2 \%$ ) in relapse. Five patients declined the offer of chemotherapy. The average benefit indicated by Adjuvant! Online in this group was $4.2 \%$ (range, $2.4 \%$ to $5.8 \%$ ) in mortality and $9.2 \%$ (range, $8 \%$ to $12 \%$ ) in relapse. Two patients for whom the MDT recommendation was to discuss chemotherapy were not referred to a medical oncologist. The average benefit in the 71 patients who received chemotherapy was $6.3 \%$ (range, $1.5 \%$ to $17.3 \%$ ) in mortality and $12.7 \%$ (range, $5.3 \%$ to $24 \%$ ) in relapse risk.

\section{Trend Over Time}

A higher proportion of node-negative patients were recommended for chemotherapy in 2008 (56 of 94; 60\%) compared with 2007 (36 of $81 ; 44 \% ; P=.04$ ).

\section{Discussion}

Nodal status remains a significant prognostic factors and a major predictor of benefit from chemotherapy for women with early-stage breast cancer. ${ }^{9}$ In node-negative patients, the prediction of chemotherapy benefit is more complex. In this survey, larger tumor size, higher grade, ER negativity, and younger age were factors that independently influenced decisions to recommend adjuvant chemotherapy, which is in keeping with current Australian guidelines. 3,8

Although it does not replace clinical judgement, Adjuvant! Online has proven valuable in the decision-making process. In 


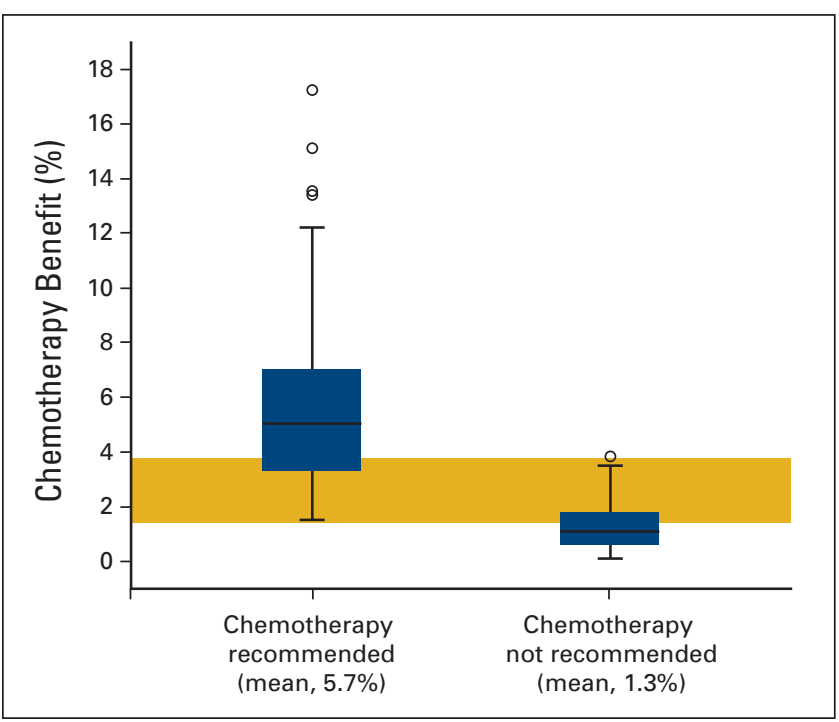

Figure 1. Mean ( \pm standard error) percentage benefit in 10-year survival per Adjuvant! Online in 175 node-negative patients who were recommended or not recommended adjuvant chemotherapy by the multidisciplinary team. The gold-shaded area represents the range of estimated chemotherapy benefit in which some but not all patients were recommended chemotherapy.

a survey from Hong Kong, members of the MDT changed their provisional recommendation for adjuvant chemotherapy after consideration of data from the program in $12 \%$ of patients; in particular, those with ER-positive, lymph node-negative disease. ${ }^{10}$ However, Adjuvant! Online may not give an accurate estimate of the benefit of systemic therapy in certain patient groups, such as those with small nodal tumor deposits and those with HER2-positive disease.

A growing body of evidence demonstrates the negative prognostic impact of small nodal tumor deposits and the benefit of systemic adjuvant therapy, even in the presence of other favorable prognostic factors. In a Dutch population-based study, micrometastasis or isolated tumor cells in the sentinel node were associated with a 9\% reduction in 5-year disease-free survival, despite favorable primary-tumor characteristics. This negative prognostic impact was reversed in patients who received adjuvant systemic therapy. ${ }^{11}$ Although the number of patients with nodal micrometastases in the current series is small, there was a clear trend toward offering chemotherapy on the basis of wider indications in this group.

HER2 overexpression is associated with more aggressive breast cancer, and recent evidence suggests this also applies to small $(<1 \mathrm{~cm})$, node-negative tumors. ${ }^{12}$ The addition of the monoclonal antibody trastuzumab to chemotherapy has a wellestablished role in HER2-positive disease, ${ }^{13}$ although its role is more controversial in patients who would otherwise not qualify for chemotherapy.

The value of the multidisciplinary meeting cannot be overestimated in interpreting patient data and discussing these nuances of evidence, in conjunction with tools such as Adjuvant! Online, to arrive at appropriate individually tailored recommendations. What magnitude of survival benefit is sufficient to make treatment-related adverse effects and inconvenience worthwhile? A number of studies that assessed the minimum benefit necessary to accept chemotherapy have shown that most patients judge a small improvement to be sufficient. Women with dependents and good social support tend to accept a smaller benefit. In one study, a 1\% increase in the likelihood of cure was judged sufficient by half of the women, and a $5 \%$ improvement was considered adequate by three quarters. ${ }^{14,15}$ On the other hand, when women who had completed chemotherapy were questioned, those with more severe adverse effects considered larger gains necessary to make their treatment worthwhile. ${ }^{16}$ Thus it is the clinician's responsibility to make a holistic assessment before recommending treatment. In this study, the threshold for recommending chemotherapy by the MDT was approximately a $2 \%$ survival benefit; however, many patients with a low estimated benefit chose not to proceed with the treatment after discussion with their medical oncologist. In fact, since the completion of this study we have introduced a distinction between a strong recommendation of chemotherapy and recommendation for a detailed discussion with a medical oncologist for patients for whom the absolute benefit is low and for whom the risks may outweigh the benefits.

This study highlights the need for better predictive tools in selecting patients with early-stage breast cancer who are likely to benefit from adjuvant chemotherapy. Recent gene-expression profiling studies have identified subsets of women who have lower risk of recurrence and do not seem to benefit from chemotherapy. In particular, this applies to women with ER-positive tumors. Several prospective trials are assessing the clinical role of these new molecular tools. ${ }^{17,18}$ Unfortunately, molecular testing is not widely available in Australia because there is currently no rebate from the Government's Pharmaceutical Benefit Scheme, which results in a nonrefundable cost to the patient of approximately 5,000 AUD. At present, we recommend these tests only to selected patients at an individual consultation as this financial dilemma may cause significant additional distress.

In conclusion, adjuvant chemotherapy is being offered increasingly over time to node-negative patients, with grade, tumor size, ER status, and younger age the most significant factors influencing chemotherapy recommendations. MDT recommendations for chemotherapy are unlikely when Adjuvant! Online-calculated survival benefits are less than $2 \%$, but after patient review, this chemotherapy threshold is higher. For some patients, risk benefit assessment is better made on the basis of clinical review by the oncologist rather than by the MDT, which emphasizes the need for personal, detailed discussion of disease management regimens.

Accepted for publication on October 18, 2010.

\section{Acknowledgment}

Supported by a clinical fellowship grant from the Cancer Institute NSW.

Authors' Disclosures of Potential Conflicts of Interest

The authors indicated no potential conflicts of interest. 


\section{Author Contributions \\ Conception and design: Elisabeth Edstrom Elder, Sally Baron Hay, Katrina Moore}

Collection and assembly of data: Elisabeth Edstrom Elder

Data analysis and interpretation: Elisabeth Edstrom Elder, Katrina Moore

Manuscript writing: Elisabeth Edstrom Elder, Sally Baron-Hay, Katrina Moore

\section{References}

1. Goldhirsch A, Ingle JN, Gelber RD, et al: Thresholds for therapies: Highlights of the St Gallen International Expert Consensus on the primary therapy of early breast cancer 2009. Ann Oncol 20:1319-1329, 2009

2. National Comprehensive Cancer Network: NCCN Clinical Practice Guidelines in Oncology: Breast Cancer. http://www.nccn.org

3. National Breast and Ovarian Cancer Centre: Clinical Practice Guidelines for the Management of Early Breast Cancer. http://www.nbocc.org.au

4. Early Breast Cancer Trialists' Collaborative Group (EBCTCG): Effects of chemotherapy and hormonal therapy for early breast cancer on recurrence and 15-year survival: An overview of the randomised trials. Lancet 365:1687-1717, 2005

5. Adiuvant! Online: Decision making tools for health care professionals. http:// www.adjuvantonline.com

6. Hassett MJ, Hughes ME, Niland JC, et al: Chemotherapy use for hormone receptor-positive, Iymph node-negative breast cancer. J Clin Oncol 26:55535560, 2008

7. American Joint Committee on Cancer: AJCC Cancer Staging Manual (ed 6). New York, Springer, 2002

8. Cancer Institute NSW: Treatment Protocols, Breast Cancer.http://www.eviq. org.au

9. Jatoi I, Hilsenbeck SG, Clark GM, et al: Significance of axillary lymph node metastasis in primary breast cancer. J Clin Oncol 17:2334-2340, 1999
Final approval of manuscript: Elisabeth Edstrom Elder, Sally BaronHay, Katrina Moore

Corresponding author: Elisabeth Edstrom Elder, MBBS, PhD, FRACS, Westmead Breast Cancer Institute, Westmead Hospital, PO Box 143, Westmead NSW 2145, Australia; e-mail: e.elder@bigpond.net.au.

DOI: 10.1200/JOP.2010.000024

10. Epstein RJ, Leung TW, Mak J, et al: Utility of a web-based breast cancer predictive algorithm for adjuvant chemotherapeutic decision making in a multidisciplinary oncology center. Cancer Invest 24:367-373, 2006

11. de Boer M, van Deurzen CH, van Dijck JA, et al: Micrometastases or isolated tumor cells and the outcome of breast cancer. N Engl J Med 361:653-663, 2009 12. Gonzalez-Angulo AM, Litton JK, Broglio KR, et al: High risk of recurrence for patients with breast cancer who have human epidermal growth factor receptor 2-positive, node-negative tumors $1 \mathrm{~cm}$ or smaller. J Clin Oncol 27: 700-706, 2009 13. Viani GA, Afonso SL, Stefano EJ, et al: Adjuvant trastuzumab in the treatment of HER-2-positive early breast cancer: A meta-analysis of published randomized trials. BMC Cancer 7:153, 2007

14. Duric V, Stockler M: Patients' preferences for adjuvant chemotherapy in early breast cancer: A review of what makes it worthwhile. Lancet Oncol 2:691-697, 2001

15. Jansen SJ, Kievit J, Nooij MA, et al: Patients' preferences for adjuvant chemotherapy in early-stage breast cancer: Is treatment worthwhile? Br J Cancer 84:1577-1585, 2001

16. Thewes B, Meiser B, Duric VM, et al: What survival benefits do premenopausal patients with early breast cancer need to make endocrine therapy worthwhile? Lancet Oncol 6:581-588, 2005

17. Zujewski JA, Kamin L: Trial assessing individualized options for treatment for breast cancer: The TAlLORx trial. Future Oncol 4:603-610, 2008

18. Cardoso F, Van't Veer L, Rutgers E, et al: Clinical application of the 70-gene profile: The MINDACT trial. J Clin Oncol 26:729-735, 2008

\section{TAKE ADVANTAGE OF ASCO'S EXCLUSIVE MEMBER-ONLY BENEFITS}

Are you experiencing the full value of ASCO membership? Along with deep discounts on meeting registrations and premier oncology publications like Journal of Clinical Oncology, ASCO also has a host of member-only benefits.

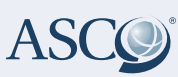

American Society of Clinical Oncology 